\title{
SIMULAÇÃO DOS COMPONENTES DA EVAPOTRANSPIRAÇÃO SOB CONDIÇÕES CLIMÁTICAS ATUAIS E DE CENÁRIOS CLIMÁTICOS FUTUROS DE AQUECIMENTO GLOBAL COM O USO DE MODELOS DE CLIMA-VEGETAÇÃO
}

\author{
GERALDO MAGELA CARDOSO ${ }^{1}$, FLÁVIO JUSTINO² \\ ${ }^{1}$ Instituto Federal do Norte de Minas Gerais, Salinas, MG, Brasil \\ ${ }^{2}$ Universidade Federal de Viçosa, Viçosa, MG, Brasil \\ geracar@ig.com.br, fjustino@ufv.br
}

Recebido Setembro de 2011 - Aceito Maio de 2013

\begin{abstract}
RESUMO
O objetivo do presente estudo foi analisar a distribuição espacial dos componentes da evapotranspiração de referência modificada (ETr) no Brasil e, posteriormente, a variabilidade temporal por meio da técnica da análise harmônica. Para isso, foi feita inicialmente uma análise de variância, fase e amplitude para o tempo presente (1980-2000) e, em seguida, feita uma projeção com o tempo futuro (2080-2100), com base no cenário A2 do Painel Intergovernamental para as Mudanças Climáticas. A equação utilizada foi a de Penman-Monteith, padronizada pela Food and Agriculture and Organization of the United Nations (FAO) 1998. As simulações foram conduzidas com o modelo regional de clima (MM5), acoplado a um modelo de vegetação potencial (MVP). Os termos aerodinâmico e radiativo apresentaram um ciclo anual que é dominante na região amazônica e Sul do Brasil, enquanto na região Sudeste o ciclo semestral tem maior destaque. As maiores variações na amplitude da ETr foram identificadas no semiárido nordestino e no extremo sul do Brasil. As mudanças na ETr, devido ao aquecimento global, foram máximas na região central do Brasil e Amazônica. Ainda foi observado que, sob condições de aquecimento global, as variações do termo aerodinâmico tornam-se mais importantes do que as variações do termo radiativo para a ETr total.
\end{abstract}

Palavra chave: Penman-Monteith, mudanças climáticas, modelagem numérica

\begin{abstract}
USE OF A REGIONAL MODEL OF CLIMATE-VEGETATION FOR ESTIMATING THE COMPONENTS OF THE REFERENCE EVAPOTRANSPIRATION UNDER CURRENT AND FUTURE CLIMATIC CONDITIONS OF GLOBAL WARMING.

The goal of this study is to analyze the spatial and temporal distribution of the components of the modified reference evapotranspiration (ETr) in Brazil. The temporal variability is investigated through the technique of harmonic analysis for two periods: present day (1980-2000) and future climate (2080-2100) based on A2 scenario of the Intergovernmental Panel on Climate Change. The method applied to evaluate the ETr was the Penman-Monteith standardized by FAO. The simulations were conducted with the regional climate model (MM5) coupled to a potential vegetation model (MVP). The results show that the aerodynamic term is dominated by the annual cycle in the Amazon region and southern Brazil, while in the southeastern the semiannual cycle has greater prominence. The largest variations in the ETr amplitude have been identified in the semi-arid part of the northeastern and in the far south of Brazil. Changes in the ETr due to global warming were highest in the central region of Brazil and the Amazon. Moreover, it has been noted that under global warming conditions changes of the aerodynamic term become more important than the variations of the radiative term in producing the ETr anomalous pattern.
\end{abstract}

Keywords: Penman-Monteith, climate changes, numerical modelling 


\section{INTRODUÇÃO}

O constante crescimento populacional representa um aumento contínuo no uso dos recursos naturais do planeta, entre eles a água. $\mathrm{O}$ uso racional desse recurso é fundamental para o destino das gerações futuras, não só para o consumo humano e animal, mas principalmente para produção de alimentos necessários à sobrevivência do homem.

A evapotranspiração é definida como o somatório da evaporação das superfícies e da transpiração das plantas (Allen et al., 1998). De acordo com o relatório publicado pela "Food and Agriculture Organization of the United Nations" (Allen et al., 1998), a evaporação depende da disponibilidade de energia na superfície evaporante, assim como do gradiente de pressão entre a superfície evaporante e o ar adjacente (Sellers, 1963). Por outro lado, a transpiração é a perda de água na forma de vapor, pelas plantas, predominantemente através das folhas, embora em plantas lenhosas seja possível também a ocorrência de pequenas perdas pelas lenticelas da casca do tronco (Amabis e Martho, 1995).

Estudos climáticos referentes às condições futuras, conduzidos com diferentes concentrações de gases de efeito estufa, apresentam substanciais variações nos campos de temperatura do ar, no ciclo hidrológico e na circulação atmosférica. Desta forma, variações nestes parâmetros poderão certamente ocasionar mudanças na evapotranspiração. Conforme Sellers (1963), a principal forçante de troca de fluxos de calor acontece entre a superfície e a atmosfera, ocasionando um gradiente de pressão de vapor entre a superfície evaporante e o ar adjacente.

Pode-se verificar que a ETr é a principal componente de perda de água para a atmosfera dentro de um balanço hídrico, e ela depende substancialmente do saldo de radiação e do déficit de pressão de vapor. Muitas pesquisas, com o intuito de se fazer uma projeção climática para o futuro, têm sido desenvolvidas, e elas se baseiam diretamente na quantidade de emissões antropogênicas de dióxido de carbono. Quatro cenários climáticos futuros têm sido desenvolvidos pelo Painel Intergovernamental de Mudanças Climáticas a saber: A1,A2,B1eB2. O cenário B1 é bem otimista. Neste cenário, no qual existe uma preocupação com o meio ambiente e com a qualidade de vida das pessoas, poderá ocorrer um aumento pouco significativo dos níveis de dióxido de carbono para em torno de $500 \mathrm{ppm}$ e a temperatura no futuro 2080-2100 poderá sofrer uma sensibilidade climática em torno de $1,5^{\circ}$. Nos cenários B2 e A1, existe pouca preocupação com o meio ambiente e com as tecnologias limpas, dessa forma os níveis de dióxido de carbono podem ficar em torno de $650 \mathrm{ppm} \mathrm{e}$ a temperatura poderá sofrer uma sensibilidade climática de $2,5^{\circ} \mathrm{C}$. Com base no cenário $\mathrm{A} 2$, existe um elevado crescimento populacional, menor preocupação com o rápido crescimento econômico e pequena preocupação ambiental. É o mais pessimista, as emissões de dióxido de carbono $\left(\mathrm{CO}_{2}\right)$ podem atingir $850 \mathrm{ppm}$ (partes por milhão).

Com base no que foi citado anteriormente, o objetivo deste trabalho foi estudar, para o período atual (1980-2000) e simular para o futuro (2080-2100), as componentes da evapotranspiração de referência modificada (ETr) com base no cenário $\mathrm{A} 2$, com os níveis de $\mathrm{CO}_{2}$ alcançando $757 \mathrm{ppm}$ e considerando as diferentes condições meteorológicas, resultantes de uma simulação climática regional a partir de um modelo de interação clima-vegetação. As simulações foram feitas por meio de avaliações das contribuições dos termos radiativo e aerodinâmico na evapotranspiração de referência modificada para os dois períodos. Pretendeu-se, ainda, investigar o ciclo sazonal da ETr, tendo como ferramenta o uso da análise harmônica.

\section{MATERIAIS E MÉTODOS}

\subsection{Estratégia do experimento numérico}

O presente estudo foi desenvolvido com base na simulação realizada por Cook e Vizy (2008), com o modelo regional MM5 (Modelo Numérico Regional) acoplado ao modelo de vegetação (MPV) de Oyama e Nobre (2004). O MM5 é um modelo de área limitada e não hidrostático. A resolução do MM5 é de 60 quilômetros com 24 níveis verticais incluindo todo o território brasileiro $\left(55^{\circ} \mathrm{S}\right.$ a $12^{\circ} \mathrm{N} \mathrm{e} 28^{\circ} \mathrm{W}$ a $\left.92^{\circ} \mathrm{W}\right)$. O MVP é bem semelhante em estrutura quando comparado com outros modelos de vegetação usados atualmente, tais como o modelo BIOME e seus descendentes (Prentice et al., 1992). O tipo de vegetação é determinado por considerar cinco parâmetros, três relacionados à temperatura do ar e dois relacionados à umidade. Esse modelo utiliza como variáveis de entrada a temperatura média do ar do mês mais frio $(\mathrm{Tc})$, em ${ }^{\circ} \mathrm{C}$; e a soma térmica acumulada $\left(\mathrm{G}\right.$, dia/mês em $\left.{ }^{\circ} \mathrm{C}\right)$; sendo $\mathrm{G}_{0}$ e $\mathrm{G}_{5}$ o tempo térmico acumulado para a temperatura basal de $0^{\circ} \mathrm{C}$ de $5^{\circ} \mathrm{C}$.

As condições iniciais e as condições de fronteira foram consideradas de acordo com o modelo CGCM3.1, do Centro Canadense e de Análise Climática e Modelagem (CCCMA), para o clima futuro (2080-2100), e a partir de dados de reanálise para a simulação do período atual (1980-2000). O clima futuro inclui o efeito do aumento de $\mathrm{CO}_{2}$ na atmosfera, chegando a 757 ppm, conforme o cenário A2 para o final do século. As análises discutidas aqui são baseadas na média de 20 anos para ambas as simulações referentes aos dias atuais e de aquecimento global. Deve-se enfatizar ainda, que o campo de evapotranspiração proposto pelo modelo não é parte das variáveis de saída do modelo MM5. Esta é a razão da investigação aqui conduzida. 
Modelo de Vegetação Potencial (MVP) utiliza asequações do balanço conforme Oyama e Nobre (2004) e usa o balanço de água e neve em separado, enquanto a evapotranpiração de referência utiliza a equação de Penman Monteith. A energia disponível depende diretamente da temperatura do ar (dada em $\left.{ }^{\circ} \mathrm{C}\right)$.

$$
\mathrm{ED}=2,265 \mathrm{~T}+67,588
$$

O acoplamento entre o MM5 e o MPV, consoante descrito por Cook e Vizy (2008), simula, sob condições de aquecimento global, a redução de até $70 \%$ da área coberta por floresta nativa na Amazônia até o final do século XXI e a sua substituição pelo bioma cerrado. Pode-se inferir, portanto, que substanciais variações na evapotranspiração e no comportamento das demais variáveis atmosféricas devem ser esperadas sob condições futuras.

\section{O European Centre For Medium-Range Weather} Forecast (ECMWF), localizado no Reino Unido, disponibiliza um produto de reanálise denominado ERA-40, o qual é um produto resultante da reavaliação das condições para a atmosfera e a superfície, compreendendo um período de 45 anos, de janeiro de 1957 a agosto de 2002. Foram utilizados vários equipamentos durante as observações, tais como radiossondas, balões, aviões, bóias, satélites. A reanálise do ERA-40 possui uma resolução vertical de 60 níveis e uma resolução horizontal de aproximadamente $110 \mathrm{~km}$. O ERA-40 produz e disponibiliza uma série de dados globais que verificam o comportamento da atmosfera e da superfície terrestre e as condições dos oceanos (Chipponelli, 2007). Os dados de reanálise foram utilizados para validação do modelo.

\subsection{Evapotranspiração de referência}

O método desenvolvido por Penman-Monteith (Equação 1) e, posteriormente, parametrizado pela FAO (Jensen et al., 1990) é conhecido atualmente como método PenmanMonteith (Allen et al., 1998). Este método é recomendado como padrão porque tem se mostrado consistente para estimar a Evapotranspiração de referência (ETr) em diferentes locais e climas, além de considerar todos os parâmetros que governam as trocas de energia e o fluxo de calor latente. Outro aspecto é que a maioria dos parâmetros pode ser medida ou estimada a partir de dados meteorológicos (Allen et al., 1998). Deve se notar que no presente estudo foram utilizados o albedo das superfícies (ex. solo, floresta) em substituição à refletividade da grama. Diante disto, define-se a ETr como sendo a evapotranspiração de referência modificada.

A equação de Penman-Monteith padrão FAO 1998 é dada a seguir:

$$
\operatorname{ETr}_{=} \frac{0,408 \Delta\left(R_{n}-G\right)+\gamma \frac{900}{T+273} U_{2}\left(e_{s}-e_{a}\right)}{\Delta+\gamma(1+0,34)}
$$

sendo,

$$
\begin{aligned}
& \mathrm{ETr}_{\text {rad }}=\frac{0,408 \Delta\left(R_{n}-G\right)}{\Delta+\gamma(1+0,34)} \\
& \mathrm{ETr}_{\text {aero }}=\frac{\gamma \frac{900}{T+273} U_{2}\left(e_{s}-e_{a}\right)}{\Delta+\gamma(1+0,34)}
\end{aligned}
$$

onde ETr é evapotranspiração de referência modificada $\left[\mathrm{mm} . \mathrm{d}^{-1}\right], \mathrm{Rn}$ o saldo de irradiância solar à superfície da cultura $\left[\mathrm{MJ} \cdot \mathrm{m}^{-2} \cdot \mathrm{d}^{-1}\right], \mathrm{G}$ é a densidade do fluxo de calor do solo $\left[\mathrm{MJ} \cdot \mathrm{m}^{-2} \cdot \mathrm{d}^{-1}\right.$ ], T a temperatura do ar média diária a $2 \mathrm{~m}$ de altura $\left[{ }^{\circ} \mathrm{C}\right], \mathrm{U}_{2}$ a velocidade do vento a 2 metros de altura $\left[\mathrm{m} . \mathrm{s}^{-1}\right], \mathrm{e}_{\mathrm{s}}$ a pressão de vapor de saturação $[\mathrm{kPa}] \mathrm{a} \mathrm{e}_{\mathrm{a}}$ a pressão parcial de vapor $[\mathrm{kPa}]$.

\subsection{Componentes de radiação}

O termo radiativo é composto pelo saldo de radiação de ondas curtas e ondas longas, temperatura do ar, fluxo de calor no solo e insolação. Devido à variação no saldo de radiação no decorrer do ano, o valor da "ETr rad" (termo radiativo da evapotranspiração) diminui nos meses de inverno, por haver menor incidência de radiação solar nesse período. Logo, de acordo com a equação (1), haverá menor ETr no inverno, assumindo que a "ETr aero" (termo aerodinâmico da evapotranspiração) não aumenta simultaneamente. A análise da componente aerodinâmica $\left(\mathrm{ET}_{\text {aero }}\right)$ depende de processos termodinâmicos como o déficit de pressão de vapor, temperatura do ar e velocidade do vento.

Variações no campo da temperatura do ar estão diretamente associadas à disponibilidade de energia ou ao saldo de radiação (Rn), o qual foi estimado por meio do modelo MM5, com base no balanço de ondas curtas (BOC) e balanço de ondas longas (BOL). Desta forma, obteve-se o Rn pela avaliação dos fluxos de radiação incidente $(\downarrow)$, refletido e emitido $(\uparrow)$, isto é:

$$
\begin{aligned}
& \mathrm{Rn}=\mathrm{BOC}+\mathrm{BOL} \\
& \mathrm{BOC}=\mathrm{S} \downarrow-\mathrm{S} \uparrow \\
& \mathrm{BOC}=\mathrm{S} \downarrow-\alpha \mathrm{S} \downarrow \\
& \mathrm{BOC}=\mathrm{S} \downarrow(1-\alpha) \\
& \mathrm{Rn}=(\mathrm{S} \downarrow-\mathrm{S} \uparrow)+(\mathrm{L} \downarrow-\mathrm{L} \uparrow) \\
& \mathrm{BOL}=\mathrm{L} \downarrow-\mathrm{L} \uparrow
\end{aligned}
$$


onde $\alpha$ é o albedo da superfície, T a temperatura da superfície $(\mathrm{K}), \sigma$ a constante de Stefan-Boltzmann $\left(5,67 \times 10^{-8} \mathrm{~W} \mathrm{~m}^{-2}\right.$ $\mathrm{K}^{-4}$ ), $\varepsilon$ a emissividade da superfície (assumida como 1 ), $\mathrm{S} \downarrow$ a radiação solar global, $\mathrm{S} \uparrow$ a radiação refletida pela superfície, $\mathrm{L} \uparrow$ a radiação de ondas longas emitida pela superfície e $\mathrm{L} \downarrow$ a radiação atmosférica.

Foi feito um estudo do comportamento da radiação de ondas longas da atmosfera, já que Galvão e Fisch (2000) demonstraram que as melhores estimativas para a região tropical foram obtidas por essa equação em detrimento das equações de Swinbank (1963), Brunt (1932), Satterlund (1979) e Bruntsaert (1975). Destaca-se que se comparada à ETo padrão FAO, a equação da evapotranspiração aqui utilizada aparece modificada, pois utiliza o albedo que depende do tipo de superfície, um coeficiente psicrométrico distinto. Isto é importante,a pois faz uso do comportamento real dos Biomas, em detrimento do uso único de grama, como sugerido pela ET0 padrão FAO.

\subsection{Análise harmônica}

A análise harmônica é uma ferramenta útil no estudo dos padrões de parâmetros meteorológicos (Aslan e Torpçu, 1994). A fim de avaliar os impactos das mudanças climáticas, a informação é usualmente necessária em escala regional e temporal. Conforme discutido por Aslan et al. (1997), a "Transformada de Fourier" ou Análise Harmônica decompõe um fenômeno periódico como uma série de funções seno e cosseno, cada uma definida por uma única amplitude, fase e variância. O ângulo de fase pode determinar a época do ano em que o valor máximo ou mínimo pode ocorrer em um dado harmônico. A análise harmônica é baseada na série de funções trigonométricas (Wilks, 1995) descrito a seguir:

$$
y_{t}=\bar{y}+\sum_{j=1}^{N} C_{j} \cos \left(\omega_{j} t-\phi_{j}\right)
$$

em que $Y_{t}$ é o valor da série no tempo " $t$ ", $\bar{y}$ a média aritmética dos valores da série, $\mathrm{C}_{\mathrm{j}}$ a j-ésima amplitude, $\mathrm{ù}_{\mathrm{j}}$ a j-ésima frequência angular, $\phi_{\mathrm{j}}$ o j-ésimo ângulo de fase, $\mathrm{N}$ o número de observações e t o tempo $\mathrm{t}$ (mês do ano).

As equações para os cálculos da amplitude dos harmônicos, segundo Emery e Thomson (1997), são:

\section{Amplitude $\left(\mathrm{C}_{\mathrm{j}}\right)$}

$$
C_{j}=\sqrt{A_{j}^{2}+B_{j}^{2}}
$$

onde $A_{j}$ é o j-ésimo coeficiente do cosseno e $B_{j}$ o j-ésimo coeficiente do seno.

Os termos $A_{j}$ e $B_{j}$ são obtidos pelas seguintes equações:

$$
\mathrm{A}_{\mathrm{j}}=\frac{2}{\mathrm{~N}} \sum_{t=1}^{N} \mathrm{y}_{\mathrm{t}} \cos \left(\frac{2 \pi \mathrm{t}}{\mathrm{N}}\right)
$$

$$
B_{j}=\frac{2}{N} \sum_{t=1}^{N} y_{t} \operatorname{sen}\left(\frac{2 \pi t}{N}\right)
$$

\section{Fase $(\phi)$}

O ângulo de fase $(\phi)$ é calculado a partir de uma das equações seguintes, dependendo do valor obtido para $\left(A_{j}\right)$ e $\left(\mathrm{B}_{\mathrm{j}}\right)$ :

$$
\begin{aligned}
& \phi=\operatorname{arctg} \frac{B_{j}}{A_{j}} \quad A_{j}>0 \text { e } B_{j}>0 \\
& \phi=\operatorname{arctg} \frac{B_{j}}{A_{j}} \pm \pi \text { ou } \pm 180^{\circ} \quad A_{j}<0 \text { e } B_{j}<0 \\
& \phi=0 \quad A_{j}=0 \text { e } B_{j}>0 .
\end{aligned}
$$

A j-ésima contribuição da cada harmônico (j) na variância total da série de dados é calculada segundo (Panofsky e Brier, 1968):

$$
j=\frac{C_{j}^{2}}{2 s^{2}}
$$

onde $\mathrm{C}_{\mathrm{j}}$ é a amplitude do harmônico e $\mathrm{S}^{2}$ a variância dos dados observados (série temporal).

A análise harmônica é um instrumento útil para caracterizar diferentes regiões climáticas e regimes de transição. O potencial da análise harmônica para classificar zonas ecoclimáticas foi discutido por Azalli e Menetti (2001).

\section{RESULTADOS E DISCUSSÃO}

\subsection{Análise harmônica da evapotranspiração para o período 1980-2000}

O clima do Brasil, sob as condições climáticas atuais, é fortemente influenciado pela Zona de Convergência Intertropical, Zona de convergência do Atlântico Sul, pelos ventos alísios, pelas montanhas dos Andes e pelos anticiclones do Atlântico e do Pacífico. Destaca-se, ainda, que a cobertura vegetal da superfície é fundamental para o estabelecimento do clima. A seguir, será apresentada uma análise do comportamento anual da "ETr" em termos do seus componentes aerodinâmicos e radiativos.

Consoante pode ser observado na Figura 1a, o ciclo anual do termo aerodinâmico da evapotranspiração é dominante em grande parte da Amazônia, região Nordeste e região Sul com uma variância explicada acima de $60 \%$. Na parte Oeste da Amazônia, em Minas Gerais, São Paulo e Mato Grosso do Sul, o ciclo semestral é dominante.

O termo radiativo depende diretamente do saldo de radiação na superfície. Na parte meridional do Brasil Central, 

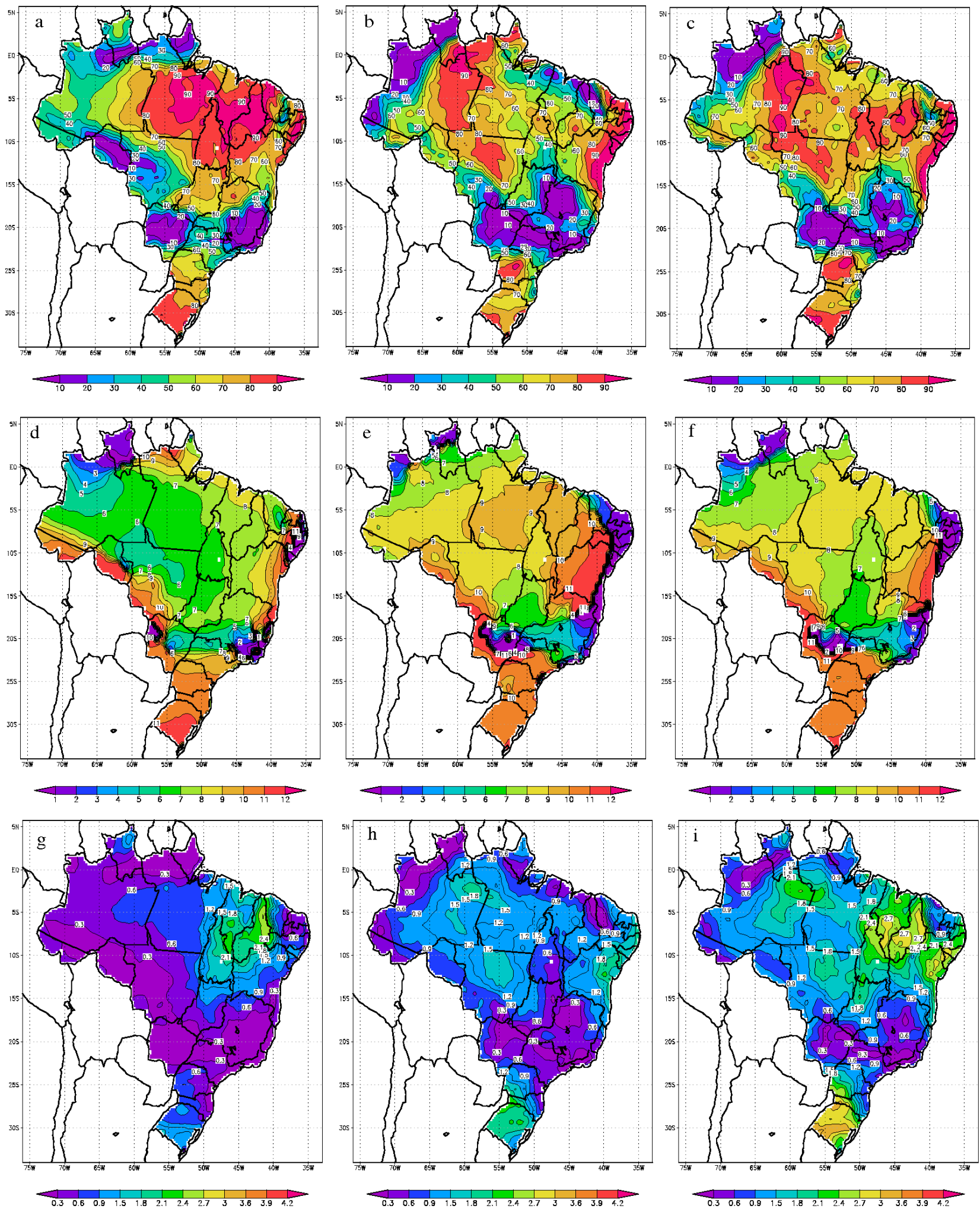

Figura 1 - (a) Variância do termo aerodinâmico (\%); (b) Variância do termo radiativo (\%); (c) Variância da ETr (\%); (d) Fase do termo aerodinâmico (meses); (e) Fase do termo radiativo (meses); (f) Fase da ETr (meses); (g) Amplitude do termo aerodinâmico (mm.dia-1); (h) Amplitude do termo radiativo (mm.dia-1); (i) Amplitude de ETr (mm.dia-1). Período 1980-2000. Dados do MM5-MVP. 
Litoral e Agreste Nordestino, bem como na região Sul, o ciclo anual explica uma variância acima de $60 \%$; ou seja, o ciclo anual é dominante nessas regiões. Em parte do Centro-Oeste, Sudeste e a parte oeste da região Amazônica, o ciclo semestral é dominante com uma variância em torno de $40 \%$.

Na Figura 1c, onde se encontra o primeiro harmônico da ETr, observa-se que o ciclo anual é dominante na região Nordeste, região Sul e em toda a parte Central do Brasil, avançando para a parte Oeste da Amazônia com variância acima de $60 \%$. No corredor de umidade composto pela região Sudeste, parte Oeste da Amazônia e pelo Mato Grosso do Sul e Sul do Mato Grosso, o ciclo semestral é dominante, com variância explicada em torno de $30 \%$. Fazendo uma análise da variância referente ao primeiro harmônico, observa-se que o termo radiativo exerce uma maior contribuição que o termo aerodinâmico, na determinação do ciclo dominante da evapotranspiração. Isso acontece porque a magnitude do saldo de radiação tem maior influência do que a ação conjunta do déficit de pressão de vapor e da velocidade do vento, na troca de fluxos de calor latente entre a superfície evaporante e a atmosfera.

No campo de fase (Figuras 1d, 1e, 1f), a máxima ETr, de acordo com o termo aerodinâmico, acontece entre os meses de setembro e novembro na parte Sul do Brasil. Na Amazônia, a fase acontece nos meses de inverno, devido à fraca atuação da Zona de Convergência InterTropical (ZCIT), que, neste período, está em sua posição mais a Norte. No litoral do Norte e Nordeste, os valores máximos do termo aerodinâmico ocorrem nos meses de agosto, setembro e outubro, devido aos ventos alísios do sudeste, juntamente com as brisas marítimas oriundas do contraste térmico entre o oceano e o continente.

$\mathrm{Na}$ Figura 1e, constata-se que a fase do primeiro harmônico na região Amazônica, Sertão do Nordeste e região Sul acontece entre agosto e novembro, por haver nessas regiões grande quantidade de radiação global associada à pouca nebulosidade (período de estiagem). No litoral do Norte e Nordeste, região centro Oeste e Sudeste, a fase acontece nos meses de janeiro a maio, devido a grande quantidade de radiação solar global que incide sobre essas regiões no período em questão. A fase dos componentes da evapotranspiração nessas regiões depende de alguns elementos meteorológicos, os quais determinam a magnitude da evapotranspiração. Os valores máximos estão relacionados ao déficit de pressão de vapor, à velocidade do vento e à quantidade de radiação global que é muito intensa nos períodos de seca (Figura 1f).

As Figuras 1g, 1h e 1i ilustram a amplitude do primeiro harmônico. Observa-se que, na região Amazônica, no Sudeste e na parte da região Sul do Brasil, a amplitude do termo aerodinâmico varia de 0,3 a $0,6 \mathrm{~mm} \cdot \mathrm{d}^{-1}$. Na região Nordeste, a amplitude varia de $1,2 \mathrm{~mm} \cdot \mathrm{d}^{-1}$ a $2,4 \mathrm{~mm} \cdot \mathrm{d}^{-1}$, devido às variações no DPV (déficit de pressão de vapor) e na magnitude dos ventos alísios. Com relação ao termo radiativo, tem-se que as maiores amplitudes são observadas na região Amazônica, Sul e parte do Nordeste. A análise da evapotranspiração, que inclui a soma dos dois termos discutidos anteriormente (Figuras $1 \mathrm{~g}$ e $1 \mathrm{~h}$ ), evidencia a ligação entre o ciclo anual dominante da ETr e o termo aerodinâmico no Nordeste, e entre a "ETr" e o termo radiativo na Amazônia, Nordeste e região Sul do Brasil. Os valores na amplitude anual da "ETr" variam de 0,3 a 2,7 mm.d $\mathrm{d}^{-1}$.

\subsection{Análise harmônica da evapotranspiração no futuro (2080-2100)}

Neste tópico, é feita uma avaliação da evapotranspiração e dos seus termos constituintes, a saber: os termos radiativo e aerodinâmico, sob um cenário de aquecimento. Na Figura 2a, o ciclo anual do termo aerodinâmico é dominante na Bacia Amazônica, com uma variância acima de $60 \%$. Tal fato difere da condição atual, na qual o ciclo anual é dominante unicamente na parte leste da bacia (Figura 1a), provavelmente devido às mudanças do vento associadas à menor rugosidade da superfície, ocasionada pela presença do cerrado substituindo a floresta nativa. O efeito do DPV também é relevante, já que o cerrado libera menor quantidade de vapor d'água para a atmosfera quando comparado com a floresta Amazônica. No Norte da Amazônia e Centro Sul do Brasil, o ciclo semianual é dominante, com variância próxima a $60 \%$. Tal fato também é verificado, em parte, na região Sul, e em boa parte da Bahia e de Minas Gerais.

Na Figura $2 b$ vê-se o ciclo anual do termo radiativo. Este é dominante no Nordeste, parte da Amazônia e Centro Sul do Brasil, tendo uma variância acima de $60 \%$. O resultado mostra a importância da mudança na vegetação, induzindo uma menor nebulosidade e, consequentemente, maior definição do ciclo sazonal das nuvens. No extremo norte e noroeste da região Amazônica e Mato Grosso predominará o ciclo semestral. Assim, o prognóstico dos modelos indica que, com o aquecimento global, a região Amazônica, a maior parte do Nordeste, e a região Sudeste, serão regidas pelo ciclo anual devido a melhor definição dos períodos seco e úmido, bem como, quente e frio. A combinação dos termos aerodinâmico e radiativo é apresentada na Figura 2c, na qual é apresentada a variância do primeiro harmônico para a "ETr". Observam-se claramente as características de ambos os componentes das ETr.

No campo de fase (Figuras 2d, 2e e 2f) verifica-se que o termo aerodinâmico é máximo entre os meses de agosto e setembro no Brasil Central e julho na Amazônia. Esse prognóstico difere do detectado no período atual, quando os 

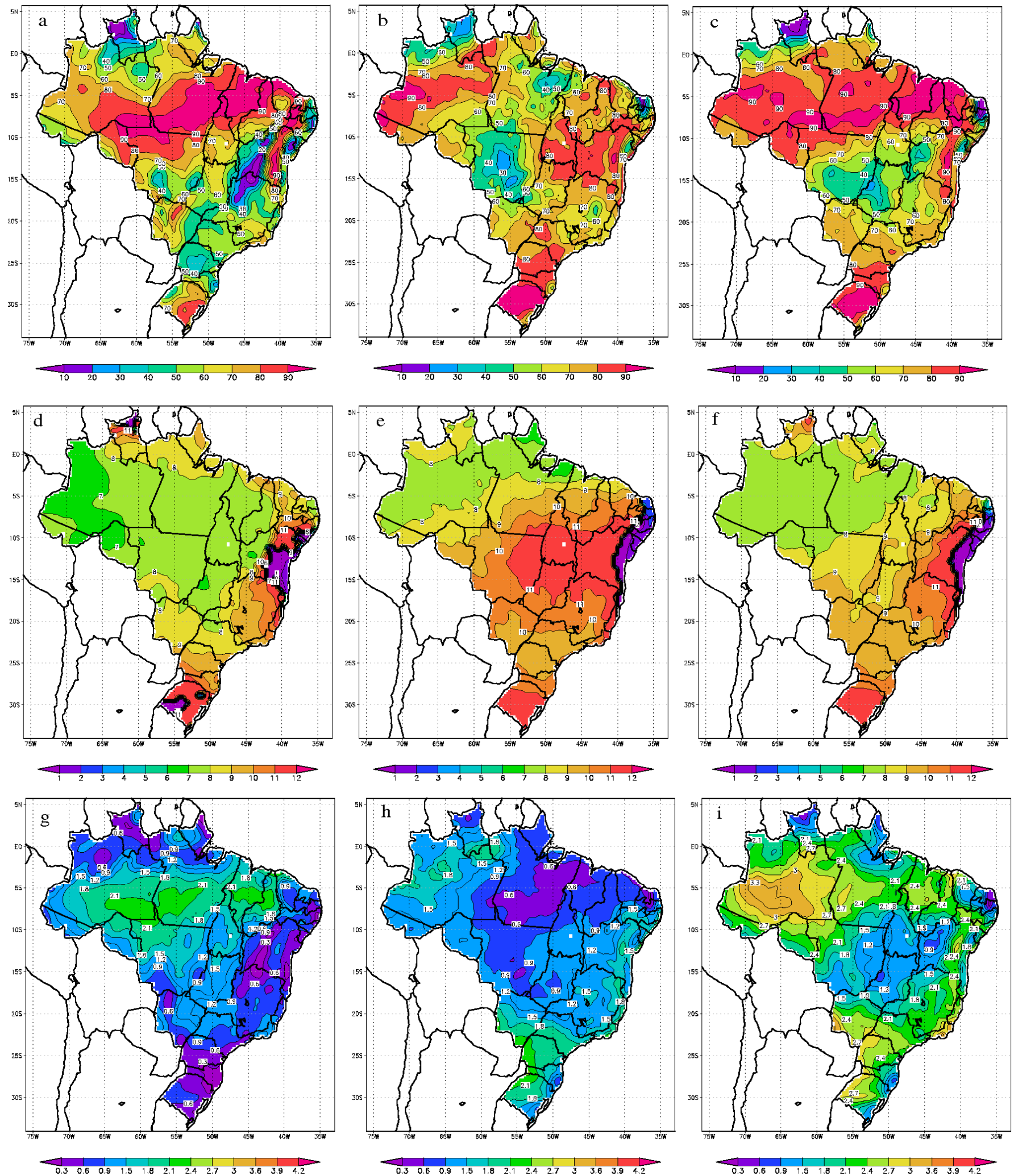

Figura 2 - (a) Variância do termo aerodinâmico (\%); (b) Variância do termo radiativo; (c) Variância da ETr; (d) Fase do termo aerodinâmico (meses); (e) Fase do termo radiativo; (f) Fase da ETr; (g) Amplitude do termo aerodinâmico (mm/dia); (h) Amplitude do termo radiativo; (i) Amplitude de ETr. Período futuro 2080-2100, cenário A2. Dados do MM5-MVP. 
máximos ocorrem dois meses antes. A causa para a mudança da fase parece estar mais relacionada ao DPV, já que o vento máximo ocorre entre maio e junho. Na Figura 2e, a fase do termo radiativo na parte Leste da Amazônia ocorre em outubro e novembro e, no litoral do Nordeste brasileiro, ocorre no mês de janeiro. No estado do Amazonas e região Centro Sul, a fase acontece de agosto a novembro. É importante notar que existem mudanças substanciais no campo de fase entre as duas simulações climáticas. Todo o cinturão compreendido entre as latitudes de $6^{\circ}$ a $20^{\circ} \mathrm{S}$ passa a ter o máximo do termo radiativo em outubro e novembro, para a simulação do período 2080-2100. Na simulação para o período atual, tal fato ocorre ao longo de vários meses. Deve-se observar ainda que, nesta região, o clima atual (futuro) é dominado pelo harmônico semianual (anual).

De acordo com a Figura 2f, o máximo da evapotranspiração na Amazônia ocorrerá nos meses de julho e agosto, e na maior parte do Brasil ocorrerá de setembro a novembro. A evapotranspiração, no futuro, sofrerá uma retração ou recuo em quase toda parte Central e Sudeste do Brasil. Todavia, pouca ou nenhuma alteração no campo de fase aparece no Nordeste em relação ao período atual (Figura $2 \mathrm{f}$ ).

As Figuras 2g, 2h e 2i indicam que, quando há um aumento na variância do primeiro harmônico, ocorrem maiores valores na amplitude. Na Figura $2 \mathrm{~g}$, verifica-se que, no Centro Sul e Litoral do Nordeste, a amplitude do termo aerodinâmico varia de 0,3 a 1,5 mm.d ${ }^{-1}$, enquanto na região Amazônica os valores da amplitude atingem $2,1 \mathrm{~mm} . \mathrm{d}^{-1}$, devido à diminuição da umidade específica no período de seca, bem como, às mudanças na vegetação da floresta tropical para o cerrado aumentando o vento naquela região.

Em comparação com o observado para o termo radiativo na condição do clima atual (Figura $2 \mathrm{~h}$ ), haverá um aumento significativo na amplitude deste termo da "ETr" no Centro-Sul. Analisando o campo de evapotranspiração (Figura 2i), percebe-se o aumento na amplitude do ciclo anual da evapotranspiração na Amazônia, Centro-Oeste e Sudeste que podem chegar a $3,3 \mathrm{~mm} \cdot \mathrm{d}^{-1}$, quando comparado às condições atuais (Figura 2i). No Sertão do Nordeste, ocorrerá uma pequena diminuição dos valores da amplitude por causa da mudança na vegetação de Caatinga para semideserto, a qual está associada ao termo radiativo devido às mudanças no albedo da superfície. Com a mudança do ciclo semianual para o anual, parte da Amazônia apresentará aumento na amplitude de até $3,3 \mathrm{~mm} \cdot \mathrm{d}^{-1}$.

A seguir é feita uma análise das diferenças entre a magnitude dos termos radiativo e aerodinâmico e da evapotranspiração para o Brasil, quanto às condições climáticas atuais e futuras. Nas Figuras 3a, 3b e 3c, o termo radiativo apresenta maior magnitude do que aerodinâmico no cálculo da evapotranspiração, em particular na porção oriental Norte e parte do Nordeste do Brasil. A contribuição do termo aerodinâmico (Figura 3a), na maior parte do território brasileiro, varia em torno de 0,6 a 1,5 mm.d ${ }^{-1}$, com exceção do Sertão do Nordeste, no que pode chegar a $3,3 \mathrm{~mm} \cdot \mathrm{dia}^{-1}$. Nesta parte do Nordeste, a velocidade do vento e o déficit de pressão de vapor são intensos, consequência do período de seca e dos ventos alísios. O termo radiativo para o presente (1980-2000), também chega a 1,5 $\mathrm{mm} \cdot \mathrm{d}^{-1}$ como conseqüência da variação na alta cobertura de nuvens e da declinação solar no decorrer do ano. No sertão do nordeste, os valores do termo radiativo podem chegar $3 \mathrm{~mm} . \mathrm{d}^{-1}$ devido à alta incidência de radiação solar na maior parte do ano nessa região.

A análise da evapotranspiração mostra que o interior dos estados do Piauí e Ceará são os que apresentam os maiores valores de evapotranspiração, podendo chegar a $5 \mathrm{~mm} \cdot \mathrm{d}^{-1}$. No Centro Sul, os valores de "ETr" chegam até $2 \mathrm{~mm} . \mathrm{d}^{-1} \mathrm{e}$, na Amazônia e litoral do Nordeste podem chegar a $3 \mathrm{~mm} \cdot \mathrm{dia}^{-1} \mathrm{em}$ relação às condições atuais.

Cook e Vizy (2008) demonstraram que as condições de umidade e seca da Amazônia estão estreitamente relacionadas às condições da superfície. Werth e Avissar (2004) identificaram ligações entre condições climáticas e processos de superfície e sub-superfície, demonstrando, que durante a estação seca, quando a precipitação total é inferior a $100 \mathrm{~mm}$, as raízes podem captar água a uma profundidade de até 3 metros, o que é suficiente para manter altas taxas de evapotranspiração da floresta, semelhante ou superiores, às da estação chuvosa.

$\mathrm{Na}$ Figura $3 \mathrm{~g}$, onde é apresentado o termo aerodinâmico da "ETr" sobre condições de aquecimento global, existe aumento em sua magnitude quando comparado ao seu correspondente para o período atual (Figura 3a), no Nordeste e na região Central da Amazônia. Tal fato é claramente notado no campo que ilustra as diferenças entre as duas simulações climáticas (Figura 3g). As anomalias positivas poderão chegar a $1,5 \mathrm{~mm} \cdot \mathrm{d}^{-1}$ na região Central da Amazônia, Bahia, Minas Gerais, Rio Grande do Norte e Paraíba. Essas anomalias positivas estão diretamente relacionadas às mudanças no campo do vento e no DPV, sendo esta última principalmente na Amazônia. Em parte da região Sul, há uma anomalia negativa que chega a $0,9 \mathrm{~mm} \cdot \mathrm{d}^{-1}$ devido a uma diminuição do DPV, o qual pode ser representado pelo aumento na umidade específica (Figura 4c).

Nas Figuras 3e e 3 h é visível o aumento significativo na magnitude do termo radiativo em toda a Amazônia, no CentroOeste, Sudeste e Sertão do Nordeste, onde os valores do termo radiativo podem atingir $3,5 \mathrm{~mm} \cdot \mathrm{d}^{-1}$. Esses valores, quando comparados aos simulados para o período atual, resultam em anomalias positivas da ordem de $2,5 \mathrm{~mm} . \mathrm{d}^{-1}$. Tais valores são intensificados porque há enfraquecimento da ZCAS nessa região (Figura 4d). Em alguns estados, como Rio Grande do Sul e 

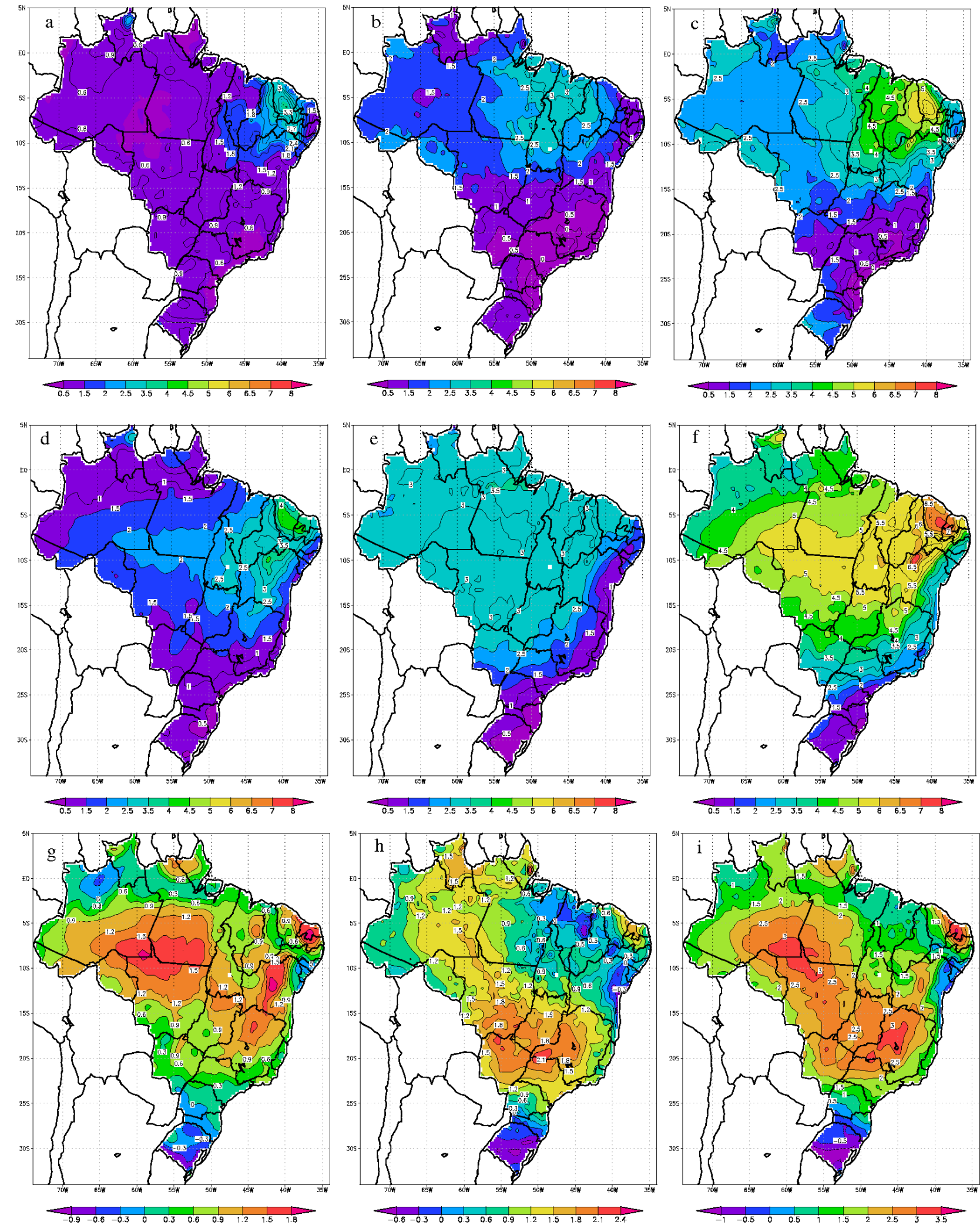

Figura 3 - (a) Magnitude dos termos radiativos - clima atual; (b) aerodinâmico - clima atual,e (c) ETr - clima atual, (d) Magnitude dos termos radiativos - clima futuro - cenário A2; (e) aerodinâmico- clima futuro - cenário A2 e (f) ETr - clima futuro - cenário A2, (g) Magnitude dos termos radiativos- diferença entre clima atual e futuro; (h) aerodinâmico - diferença entre clima atual e futuro e (i) ETr- diferença entre clima atual e o futuro. 

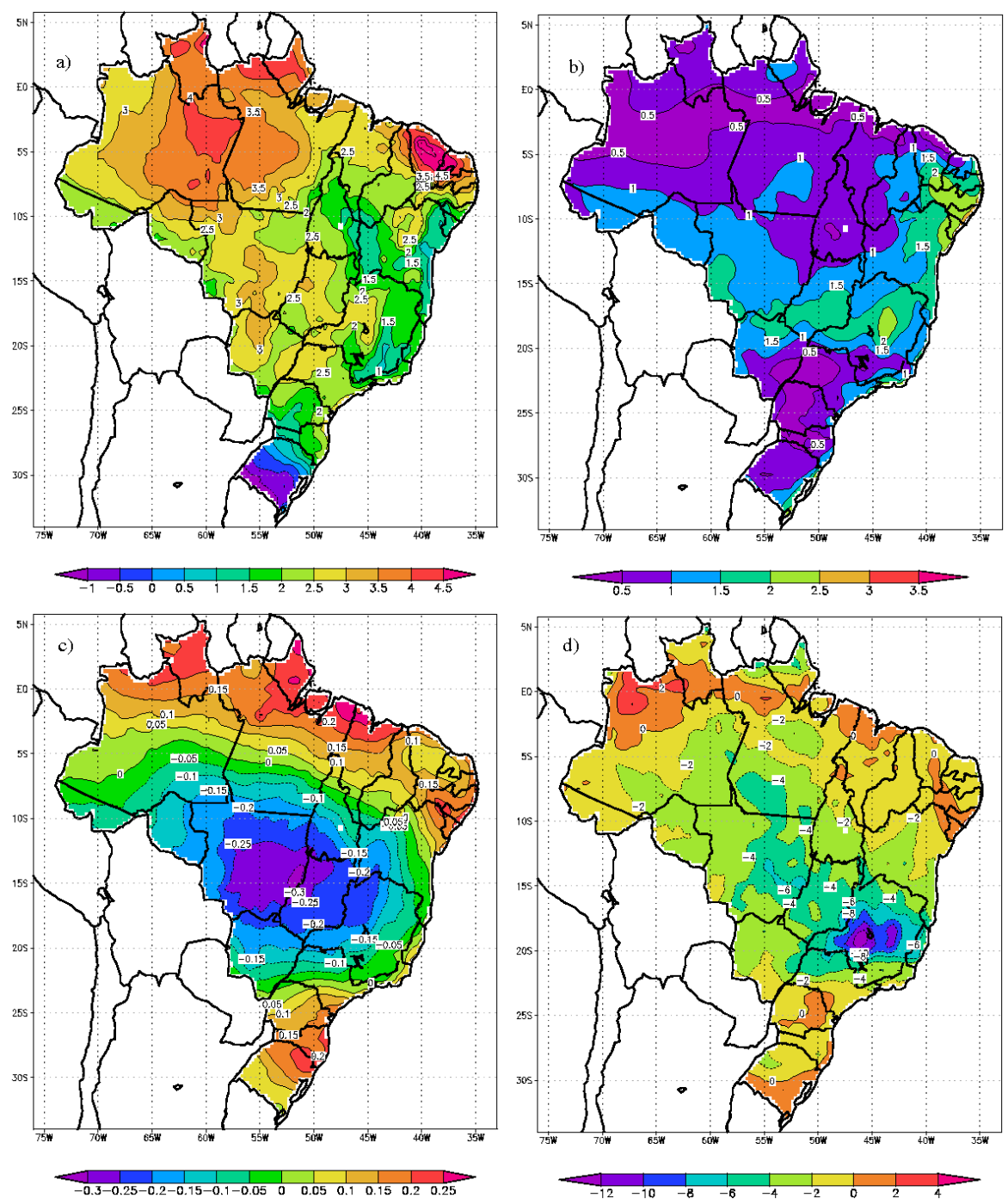

Figura 4 - Anomalias na magnitude da média anual de temperatura $\left({ }^{\circ} \mathrm{C}\right)$, velocidade do vento (m.s-1), umidade específica (10-2 kg.kg-1) e precipitação (mm.d-1), entre o presente (1980-2000) e o futuro (2080-2100). Dados do MM5-MVP.

Paraná, ocorrem anomalias negativas por causa do aumento na cobertura de nuvens nessas regiões.

No campo da evapotranspiração de referência (Figuras 3f e 3i), há um aumento significativo na Amazônia e Sertão do Nordeste, com valores que podem atingir 6-7 mm.dia ${ }^{-1}$ (Figura 3f). Acerca das condições atuais, estas regiões apresentam valores da ordem de 3-4 mm.d ${ }^{-1}$ (Figura 3c). Com aumento dos gases de efeito estufa no futuro (2080-2100) e, consequente, mudança na vegetação, principalmente na região Amazônica, as anomalias de "ETr" podem chegar a $3 \mathrm{~mm} \cdot$ dia $^{-1}$ (Figura 3i). No Norte da Amazônia, com a manutenção da floresta, há uma anomalia positiva de menor intensidade na magnitude da evapotranspiração, quando comparada ao sul da bacia Amazônica. Isso se deve à manutenção do vapor d'água pelas florestas, o que contribui para a diminuição do déficit de pressão de vapor.

\section{CONCLUSÕES}

De acordo com os resultados obtidos tem-se ainda que: (1) Sob condições atuais, o modelo apresenta bom desempenho do ciclo anual quando comparado aos dados do ERA-40; (2) os termos aerodinâmico e radiativo têm o ciclo anual que é dominante na região Amazônica e Sul do Brasil, enquanto na região Sudeste o ciclo semestral é dominante; (3) as maiores variações na amplitude da "ETr" ocorrem no semiárido nordestino e no extremo Sul do Brasil, (4) as mudanças na "ETr" devido ao aquecimento global, serão máximas na região central do Brasil e Amazônica; (5) em condições futuras o ciclo anual será dominante na maior parte do Brasil, o que indica uma estação seca e uma estação chuvosa bem definidas; (6) Com as condições climáticas do presente 
(1980-2000), a evapotranspiração no Brasil é caracterizada pela separação meridional e zonal em termos de ciclo dominante. Com as simulações para o futuro (2080-2100), num cenário de aquecimento global, a separação meridional e zonal da evapotranspiração no Brasil deixa de existir e há uma mudança substancial no ciclo da evapotranpiração, na região ocidental da Amazônia e regiões Sudeste e Centro Oeste. No referente às condições de aquecimento global, as variações do termo aerodinâmico tornar-se-ão mais expressivas do que as variações do termo radiativo, para a determinação da "ETr" total.

A grande contribuição deste trabalho para a ciência é que, através de um modelo regional de clima MM5, envolvendo várias variáveis meteorológicas, se acopla a um Modelo de Vegetação Potencial que consegue diagnosticar qual o tipo de vegetação potencial entra em equilíbrio com um determinado clima. É possível, dentro de um cenário de aquecimento global, fazer simulações de variabilidade e sensibilidade da vegetação potencial para o período futuro (2080-2100), com base em um cenário $\mathrm{A}_{2}$ do IPCC com níveis de $\mathrm{CO}_{2}$ atingindo $757 \mathrm{ppm}$.

\section{AGRADECIMENTO}

Os autores do trabalho agradecem imensamente à disponibilidade dos dados, proporcionada pelos Dr. Kerry Cook and Edward Vizy da Universidade do Texas em Austin. À CAPES, pelo fornecimento de bolsa de estudo.

\section{REFERÊNCIAS}

ALLEN, R. G.; PEREIRA, L.S.; RAES,D.; SMITH,M.; Crop evapotranspiration: guidelines for computing crop water requirements. Rome: FAO, 1998.300 P. (Irrigation and Drainage, $\mathrm{N}^{\mathrm{o}} 56$ ).

AMABIS, MARTHO. Biologia dos organismos. Volume 2. São Paulo, Editora Moderna, 1995.

ASLAN, Z.; TOPÇU, S. Seasonal variation of surface fluxes and atmospheric interaction in Istanbul. Lisbon, Portugal, 1994.

ASLAN, Z.; OKCU, D.; KARTAL, S. Harmonic analysis of precipitation, pressure and temperature over turkey. II Nuovo Cimento, v. 20, p. 595-605, 1997.

AZALLI, S.; MENETTI, M. Mapping vegetation-soil-climate complexes in Southern Africa using temporal fourier analysis of Noaa-Avhrr Ndvi Data. International Journal of Remote Sensing, v. 21, n. 5, p. 973-996, 2001.

BRUNT, D. Notes on radiation in the atmosphere. Quarterly Journal of The Royal Meteorological Society, v. 58, p. 389-420, 1932.
BRUNTSAERT, W. Evaporation into the atmosphere. Reidel Publishing Company, 1975. 299 p.

CHIPPONELLI, L. I. P. Comparação de produtos de precipitação e radiação solar incidente para a América do Sul: dados observados e reanálises. 2007. 75 p. Dissertação (Mestrado em Meteorologia Agrícola) - Universidade Federal de Viçosa, Viçosa, MG.

COOK, K.; VIZY, E. K. Effects of twenty-first-century climate change on the amazon rain forest. Journal of Climate, $v$. 21, n. 3, p. 542, 2008.

EMERY, W.; THOMSON, R. Data analysis methods in physical oceanography. Oxford: Pergamon/Elsevier Science, 1997. $643 \mathrm{p}$.

GALVÃO, J. A.; FISCH, G. Balanço de radiação em área de pastagem na Amazônia. Revista Brasileira de Agrometeorologia, v. 8, n. 1, p. 1-10, 2000.

JENSEN, M. E.; BURMAN, R. D.; ALLEN, R. G. Evapotranspiration and irrigation water requirements. New York: ASCE, 1990. 360 p. (Manuals and Reports on Engineering Practices, 70).

OYAMA, M. D. E.; NOBRE, C.A. A simple potential vegetation model for coupling with the Simple Biosphere Model. Brazilian Journal of Meteorology, v. 19, p. 204-216, 2004.

PANOFSKY, H. A.; BRIER, G. W. Some applications of statistics to meteorology. Pennsylvania: University Park, 1968. $224 \mathrm{p}$.

PRENTICE, I. C.; CRAMER, W.; HARISON, S. P.; LEEMANS, R.; MONSERUD, R. A.; SOLOMON, A. A global biome model based on physiology and dominance, soil properties and climate. Journal of Biogeography, v. 19, n. 2, p. 117134, 1992.

SWINBANK, W. C. Long-wave radiation from clear skies. Quarterly Journal of The Royal Meteorological Society, v. 89, p. 339-348, 1963.

SATTERLUND, D. R. An improved equation for estimating long-wave radiation from the atmosphere. Water Resources Res., 15: 1649-1650, 1979.

SELLERS, W. Potential evapotranspiration in arid regions. Journal of Climate, v. 3, p. 98-105, 1963.

WERTH, D.; AVISSAR, R. The regional evapotranspiration of the amazon. Journal of Hydrometeorology, v. 5, p. 100-109, 2004.

WILKS, D. S. Statistical methods in the atmospheric sciences: an introduction. San Diego, California: Academic Press, 1995. $467 \mathrm{p}$. 